\title{
Echocardiographic assessment in patients with atrial fibrillation (AF) and normal systolic left ventricular function before and after catheter ablation: If AF begets AF, does pulmonary vein isolation terminate the vicious circle?
}

\author{
Aleksandra Liżewska-Springer, Alicja Dąbrowska-Kugacka, Ewa Lewicka, \\ Tomasz Królak, Łukasz Drelich, Dariusz Kozłowski, Grzegorz Raczak \\ Department of Cardiology and Electrotherapy, Medical University of Gdansk, Poland
}

\begin{abstract}
Background: Radiofrequency catheter ablation (RFCA) of atrial fibrillation (AF) can be curative. There are conflicting data on whether $A F$ associated atrial and ventricular structural remodeling reverses after ablation. The aim of this study was to evaluate the hemodynamic effect of AF ablation in patients with preserved left ventricular ejection fraction (LVEF).

Methods: Forty three AF patients were studied (aged $56 \pm 11$ years; 29 male, 23\% persistent AF, $L V E F \geq 50 \%)$ in whom RFCA was performed. Echocardiographic evaluation of atrial and ventricular diameters, volumes and strain imaging by two-dimensional speckle tracking were performed before and at least 6 months after RFCA. Nine patients had AF during baseline examination.

Results: A significant decrease in the left (LA) and right (RA) atrial volume and an increase in the $L A$ strain were observed $15 \pm 7$ months after RFCA. In the subgroup with baseline sinus rhythm, the increment in LA strain was only borderline significant. An increase in $R A$, right ventricular $(R V)$ and Biatrial strain was noticed $(p<0.05)$. LVEF and global longitudinal strain of the left ventricle $(L V)$, however, did not improve substantially.

Conclusions: Radiofrequency catheter ablation of $A F$ in patients with preserved $L V$ systolic function results in significant improvement in $R A$ and $R V$ function with a substantial reduction in LA and $R A$ size. No deleterious impact of AF ablation on LA function was revealed. (Cardiol J 2020; 27, 2: 126-135)

Key words: atrial fibrillation, catheter ablation, pulmonary vein isolation, cardiac remodeling, echocardiography
\end{abstract}

\section{Introduction}

Radiofrequency catheter ablation (RFCA) has become a common treatment for atrial fibrillation (AF). RFCA improves symptoms and quality of life by reducing arrhythmia burden $[1,2]$. Nevertheless, AF ablation outcome regarding cardiac remodeling is still questionable. Imaging analysis such as echocardiography reveals that RFCA changes AF- induced remodeling which affects both the atrium and ventricle. It is known that RFCA improves ejection fraction $(\mathrm{EF})$ in patients with heart failure and significantly decreases left atrial (LA) size, especially after successful restoration of sinus rhythm (SR) [3, 4]. However, despite both functional and structural changes after RFCA having been widely studied the impact of $\mathrm{AF}$ ablation on myocardial muscle has not yet been determined. First, in patients with preserved left ventricular (LV) function from previous studies demonstrated

Address for correspondence: Dr. Alicja Dąbrowska-Kugacka, Department of Cardiology and Electrotherapy, Medical University of Gdansk, ul. Dębinki 7, 80-210 Gdańsk, Poland, tel: +48 5834939 10, e-mail: adabrowska@gumed.edu.pl Received: 27.11.2018 Accepted: 21.01.2019 
no change in EF [5, 6]. However, left ventricular ejection fraction (LVEF) estimation does not reflect subtle changes in $\mathrm{LV}$ and therefore the effect of $\mathrm{AF}$ ablation on LV function is still uncertain. Second, it is not known whether AF ablation results in any change in the right ventricular (RV) size and function. Finally, the effect of RFCA on both LA and right atrial (RA) functional changes remains uncertain. Therefore, it is controversial to what extent RFCA can stop AF induced cardiac remodeling. Recently two-dimensional speckle tracking echocardiography (2D-STE) has been widely accepted as an innovative method to evaluate the function and remodeling of the heart muscle. In addition, 2D-STE correlates with cardiac magnetic resonance imaging results $[7,8]$.

The aim of this mono-center prospective study was to evaluate the effect of AF ablation on hemodynamic using 2D-STE.

\section{Methods}

\section{Patient enrollment}

Patients with paroxysmal or persistent nonvalvular $\mathrm{AF}$ who underwent RFCA during the period between January 2014 and December 2017 were considered for enrollment. The exclusion criteria were as follows: LVEF $<50 \%$ before RFCA, clinically significant valve disease or prosthetic valve, unstable coronary artery disease, bundle branch block, severe LV hypertrophy, uncontrolled thyroid or pulmonary disease in anamnesis. Finally, data for 43 consecutive patients were used for analyses in this study. The study was approved by local ethics committee (ethical approval reference number: NKBBN/505/2017) and all patients gave their written informed consent before procedures.

\section{Electrophysiologic study and catheter ablation}

One RFCA session was performed in 40 patients, and 3 patients needed a second session due to $\mathrm{AF}$ recurrences. The procedure was performed under conscious sedation using midazolam and fentanyl. A diagnostic catheter was positioned via the left femoral vein into the coronary sinus and the region of His bundle. Then via the right femoral vein a double transseptal puncture was performed and an ablation catheter (ThemoCool, SmartTouch, Biosense-Webster, CA, USA) and a multipolar catheter (Lasso, Biosense-Webster, CA, USA) were introduced into the LA. A three-dimensional electroanatomical map was created using the Carto-3 system (Biosense-Webster, CA, USA). All patients underwent extensive encircling pulmonary vein isolation (PVI) using a maximum power of 20-30 Watts. During procedure the activated clotting time was controlled and maintained above $300 \mathrm{~s}$. Bi-directional electrical PVI was considered the endpoint of ablation and was confirmed by the complete disappearance of pulmonary vein potentials recorded by circular catheter and absence of veno-atrial conduction during intra-pulmonary vein pacing. A cavo-tricuspid isthmus block line was created in $1(2 \%)$ patient who had documented atrial flutter, apart from $\mathrm{AF}$.

\section{Echocardiographic study}

Transthoracic echocardiography was performed before and at least 6 months after RFCA using a Vivid E9 (General Electric Medical Health). Two-dimensional (2D) and Doppler measurements were obtained according to recommendations for cardiac chamber quantification [9]. Cine loops from three standard apical views (4-, 3-, and 2-chamber) were recorded for off-line analysis (EchoPac 201, GE).

Left atrial minimal and maximal volumes were calculated using the Simpson rule from 4- and 2 -chamber views. RA minimal and maximal volumes were calculated using a single-plane method of disks from 4-chamber view. LA emptying fraction (LAEF) was calculated according to the formula: (maximum - minimum LA volume)/maximum LA volume $\times 100$. Both, LA and RA volumes were indexed to body surface area (LAVI and RAVI).

\section{Two-dimensional speckle tracking echocardiography}

Left ventricular global longitudinal peak strain (LV-GLS) was calculated according to the recommendations for cardiac chamber quantification [9]. Measurements were made in three standard apical views and expressed as a mean value.

Furthermore LA, RA and RV strain measurements were performed according to the consensus document standardizing deformation imaging [10]. The measurements for LA were obtained at each myocardial segment of the apical 4-chamber view and for RA and RV of the RV-focused 4-chamber view. The endocardial wall borders were traced manually and a region of interest was created automatically by the software (EchoPac). Segments with inadequate tracking were excluded from analysis. The QRS wave was used as the zero point in both SR and AF rhythm. The average value from all 6 segments of LA or RA during the entire cardiac cycle was used for the final value of LA or RA positive peak strain. Additionally, 
Table 1. Clinical characteristics of the whole examined group and with division according to heart rhythm during the echocardiographic examination before arrhythmia ablation.

\begin{tabular}{lcccc}
\hline & $\begin{array}{c}\text { Total } \\
(\mathbf{n}=43)\end{array}$ & $\begin{array}{c}\text { SR group } \\
(\mathbf{n}=34)\end{array}$ & $\begin{array}{c}\text { Group with } \\
\text { baseline AF (n = 9) }\end{array}$ & $\begin{array}{c}\text { *P value between } \\
\text { SR and AF group }\end{array}$ \\
\hline Age & $56 \pm 11$ & $54 \pm 12$ & $60 \pm 9$ & 0.16 \\
Sex (male) & $29(85 \%)$ & $22(65 \%)$ & $7(78 \%)$ & 0.73 \\
Hypertension & $22(51 \%)$ & $17(50 \%)$ & $5(56 \%)$ & 0.94 \\
Hypercholesterolemia & $14(33 \%)$ & $10(29 \%)$ & $4(45 \%)$ & 0.65 \\
Type 2 diabetes non-insulin dependent & $2(5 \%)$ & $1(4 \%)$ & $1(12 \%)$ & 0.85 \\
Stable CAD & $2(5 \%)$ & $2(6 \%)$ & None & 0.88 \\
Obesity & $2(5 \%)$ & $2(6 \%)$ & None & 0.88 \\
Persistent AF & $10(23 \%)$ & $5(15 \%)$ & $5(56 \%)$ & $<0.05$ \\
Redo-PVI & $3(7 \%)$ & $3(9 \%)$ & None & 0.85 \\
CTI ablation & $1(2 \%)$ & $1(4 \%)$ & None & 0.47 \\
Amiodarone & $6(14 \%)$ & $5(15 \%)$ & $1(12 \%)$ & 0.79 \\
Sotalol & $9(21 \%)$ & $7(21 \%)$ & $2(23 \%)$ & 0.72 \\
I C anti-arrhythmic drugs & $13(30 \%)$ & $11(32 \%)$ & $2(23 \%)$ & 0.86 \\
No anti-arrhythmic drugs & $17(40 \%)$ & $11(32 \%)$ & $6(67 \%)$ & 0.14 \\
Beta-blocker (another than sotalol) & $16(37 \%)$ & $13(38 \%)$ & $3(33 \%)$ & 0.91 \\
ACEl/AT II & $10(23 \%)$ & $8(24 \%)$ & $2(22 \%)$ & 0.72 \\
Statin & $17(40 \%)$ & $14(41 \%)$ & $3(33 \%)$ & 0.96 \\
AF recurrence & $11(26 \%)$ & $10(29 \%)$ & $1(12 \%)$ & 0.49 \\
\hline
\end{tabular}

Age values are mean \pm standard deviation. ${ }^{*}$ Chi-squared test with Yates correction. SR — sinus rhythm; AF — atrial fibrillation; CAD — coronary artery disease; $\mathrm{PVI}$ - pulmonary vein isolation; CTI — cavo-tricuspid isthmus; ACEI — angiotensin-converting enzyme inhibitor; AT II — angiotensin II receptor blocker

the endocardial border of LA and RA free wall was traced to obtain biatrial positive peak strain (excluding atrial septum). Similarly, as with atrial analysis, RV negative peak strain was calculated. The region of interest included both the RV free wall and interventricular septum.

\section{Follow-up}

All patients were monitored by 12 -lead electrocardiography (ECG) and 24-h Holter recording in an outpatient Arrhythmia Clinic at averagely 3, 6 and 12 months after ablation. Anti-arrhythmic therapy was prescribed in patients with easily inducible AF. In cases of symptoms suggestive of AF immediate ECG or Holter recording was performed. Echocardiography was repeated at least 6 months after RFCA. The ablation was considered successful if during the follow-up there was no recurrence of symptoms suggestive of $\mathrm{AF}$, no $\mathrm{AF}$ recorded on standard $\mathrm{ECG}$ and no AF lasting $>30$ s on Holter monitoring, after a blanking period of 3 months.

\section{Statistical analysis}

Statistical analysis was performed using the licensed Statistica 13 software (Statsoft Poland).
Continuous variables are presented as mean \pm standard deviations (SD) or median-with first and third interquartile range. Statistical significance was defined as $\mathrm{p}<0.05$. The Shapiro-Wilk test was used to estimate the distribution. The $\chi^{2}$ test with Yates correction was used for nonparametric data and the two-sample t-test for continuous variables. Comparison of the echocardiographic parameters before and after RFCA was performed by the paired sample t-test or Wilcoxon signed-rank test where appropriate. Two-way ANOVA with replication was used for comparison of the echocardiographic parameters before and after RFCA in the SR and $\mathrm{AF}$ group. Intra- and interobserver variability for strain measures were determined in 10 randomly selected patients and the variability was calculated as an absolute difference (in \%) divided by the mean of the repeated measures.

\section{Results}

\section{Baseline characteristics and follow-up}

Baseline clinical characteristics of the patients enrolled $(n=43)$ is listed in Table 1 . All analyzed patients had a history of AF of at least 1-year in 
Table 2. Echocardiographic parameters in the whole group examined $(n=43)$ before and after radiofrequency catheter ablation (RFCA).

\begin{tabular}{|c|c|c|c|}
\hline & Baseline & After RFCA & $\mathbf{P}$ \\
\hline Heart rate & $57 \pm 16$ & $67 \pm 10$ & 0.51 \\
\hline Interventricular septum [mm] & $10(9-12)$ & $11(9-11)$ & $0.55^{*}$ \\
\hline Posterior wall [mm] & $9 \pm 2$ & $9 \pm 1$ & 0.63 \\
\hline LV end-diastolic diameter [mm] & $50 \pm 5$ & $49 \pm 5$ & 0.49 \\
\hline LV end-systolic diameter [mm] & $32 \pm 5$ & $32 \pm 5$ & 0.38 \\
\hline LV end-diastolic volume [mL] & $88(80-108)$ & $91(82-103)$ & $0.44^{*}$ \\
\hline LV end-systolic volume [mL] & $37(30-45)$ & $35(30-44)$ & $0.69 *$ \\
\hline LVEF [\%] & $60 \pm 7$ & $62 \pm 6$ & 0.15 \\
\hline LV-GLS [\%] & $-18 \pm 4$ & $-19 \pm 3$ & 0.05 \\
\hline RV end-diastolic area $\left[\mathrm{cm}^{2}\right]$ & $18(17-22)$ & $18(15-21)$ & $<0.01^{*}$ \\
\hline TAPSE $[\mathrm{mm}]$ & $22 \pm 5$ & $24 \pm 3$ & 0.01 \\
\hline $\mathrm{s}^{\prime} \mathrm{RV}[\mathrm{m} / \mathrm{s}]$ & $0.12(0.12-0.15)$ & $0.14(0.13-0.15)$ & $0.10^{*}$ \\
\hline RV strain [\%] & $-19(-16 ;-23)$ & $-23(-20 ;-26)$ & $<0.05^{*}$ \\
\hline LA diameter $[\mathrm{mm}]$ & $39 \pm 4$ & $39 \pm 4$ & 0.31 \\
\hline LA min. volume $[\mathrm{mL}]$ & $47 \pm 21$ & $38 \pm 16$ & $<0.01$ \\
\hline LA max. volume $[\mathrm{mL}]$ & $83 \pm 23$ & $73 \pm 20$ & $<0.01$ \\
\hline LAVI $\left[\mathrm{mL} / \mathrm{m}^{2}\right]$ & $41 \pm 12$ & $37 \pm 11$ & 0.01 \\
\hline LAEF [\%] & $44 \pm 14$ & $49 \pm 11$ & 0.07 \\
\hline LA strain [\%] & $23 \pm 8$ & $27 \pm 7$ & $<0.001$ \\
\hline $\mathrm{RA}$ min. volume $[\mathrm{mL}]$ & $34 \pm 18$ & $26 \pm 12$ & $<0.0001$ \\
\hline RA max. volume $[\mathrm{mL}]$ & $56(48-68)$ & $49(41-60)$ & $0.001^{*}$ \\
\hline $\mathrm{RAVI}\left[\mathrm{mL} / \mathrm{m}^{2}\right]$ & $27(24-32)$ & $24(19-31)$ & $<0.001 *$ \\
\hline RA strain [\%] & $27 \pm 11$ & $35 \pm 10$ & $<0.001$ \\
\hline Biatrial strain [\%] & $19 \pm 9$ & $26 \pm 8$ & $<0.0001$ \\
\hline$E[\mathrm{~m} / \mathrm{s}]$ & $0.7 \pm 0.2$ & $0.7 \pm 0.2$ & 0.84 \\
\hline $\mathrm{A}[\mathrm{m} / \mathrm{s}]$ & $0.6 \pm 0.2$ & $0.6 \pm 0.1$ & 0.21 \\
\hline $\mathrm{e}^{\prime}$ mean $[\mathrm{cm} / \mathrm{s}]$ & $1 \pm 0.4$ & $1 \pm 0.3$ & 0.16 \\
\hline $\mathrm{E} / \mathrm{e}^{\prime}$ & $7(6-9)$ & $7(6-10)$ & $0.43^{*}$ \\
\hline
\end{tabular}

Values are mean \pm standard deviation or median (first-third interquartile range) when normal distribution was not achieved. $P$ value: $t$-test for related samples and where appropriate *Wilcoxon signed-rank test.

LV - left ventricle; LVEF — LV ejection fraction; LV-GLS - LV global longitudinal peak strain; RV — right ventricle; TAPSE- tricuspid annular plane systolic excursion by M-mode; s' RV - peak systolic velocity of tricuspid annulus by spectral tissue Doppler; LA - left atrium; min. minimum; max. - maximum; LA — left atrial; LAVI — LA volume index; LAEF — LA emptying fraction; RA — right atrium; RAVI — RA volume index; E - early transmitral flow velocity by pulsed wave Doppler; A — late transmitral flow velocity by pulsed wave Doppler; $e^{\prime}$ — early diastolic mitral annulus velocity by spectral tissue Doppler

duration or longer (mean $4 \pm 3$ years). During the echocardiographic examination before RFCA $34(79 \%)$ patients were in SR (SR group), while $9(21 \%)$ had AF (AF group). A total of $28(65 \%)$ patients took antiarrhythmic drugs before AF ablation, $26(60 \%)$ continued taking them after the procedure ( 7 of them discontinued between 2 and 6 months). All patients had adequately controlled hypertension (blood pressure $<140 / 90 \mathrm{mmHg}$ ). The average number of antihypertensive drugs per patient was 1.1. There were no substantial differences in clinical characteristics such as age, sex, comorbidities, or pharmacotherapy before RFCA between the two groups. Only persistent AF was more frequent in the group with baseline $\mathrm{AF}$.

Successful PVI was completed in all patients; 3 (7\%) needed a second session; a cavo-tricuspid isthmus block line was created in $1(2 \%)$ patient with documented atrial flutter. During a $15 \pm$ \pm 7 month follow up, AF recurred in 11 (26\%) patients, 10 of them had SR during baseline echo examination.

\section{Echocardiographic findings}

Echocardiographic results are summarized in Tables 2 and 3. 
Table 3. Comparison of the subgroup with sinus rhythm (SR group) and atrial fibrillation (AF group) during the echocardiographic examination before radiofrequency catheter ablation (RFCA) - selected parameters.

\begin{tabular}{|c|c|c|c|c|c|c|c|}
\hline \multirow[t]{2}{*}{ Variable } & \multicolumn{2}{|c|}{$\begin{array}{l}\text { SR group } \\
(\mathrm{n}=34)\end{array}$} & \multicolumn{2}{|c|}{$\begin{array}{c}\text { AF group } \\
(n=9)\end{array}$} & \multirow[t]{2}{*}{$\begin{array}{c}\text { ^P value } \\
\text { (SR group vs. } \\
\text { AF group) }\end{array}$} & \multicolumn{2}{|c|}{$\begin{array}{c}\text { \#P value } \\
\text { (baseline vs. } \\
\text { after RFCA) }\end{array}$} \\
\hline & Baseline & $\begin{array}{l}\text { After } \\
\text { RFCA }\end{array}$ & Baseline & $\begin{array}{l}\text { After } \\
\text { RFCA }\end{array}$ & & $\underset{\text { group }}{\text { SR }}$ & $\underset{\text { group }}{\text { AF }}$ \\
\hline Heart rate & $62 \pm 10$ & $68 \pm 10$ & $87 \pm 18$ & $65 \pm 10$ & $<0.0001$ & $<0.01$ & $<0.01$ \\
\hline LVEF [\%] & $61 \pm 6$ & $61 \pm 7$ & $55 \pm 11$ & $63 \pm 3$ & $<0.01$ & 0.91 & 0.06 \\
\hline LV-GLS [\%] & $-19 \pm 3$ & $-19 \pm 4$ & $-14 \pm 4$ & $-20 \pm 2$ & $<0.0001$ & 0.91 & $<0.01$ \\
\hline TAPSE $[\mathrm{mm}]$ & $23 \pm 4$ & $24 \pm 3$ & $16 \pm 2$ & $23 \pm 3$ & $<0.0001$ & 0.60 & $<0.0001$ \\
\hline RV strain [\%] & $-21 \pm 5$ & $-23 \pm 5$ & $-15 \pm 3$ & $-22 \pm 2$ & $<0.01$ & $<0.01$ & $<0.0001$ \\
\hline LA min. volume $[\mathrm{mL}]$ & $43 \pm 17$ & $38 \pm 17$ & $65 \pm 24$ & $40 \pm 15$ & $<0.01$ & 0.12 & $<0.01$ \\
\hline LA max. volume [mL] & $82 \pm 22$ & $72 \pm 20$ & $87 \pm 29$ & $79 \pm 19$ & 0.75 & $<0.01$ & 0.31 \\
\hline $\mathrm{LAVI}\left[\mathrm{mL} / \mathrm{m}^{2}\right]$ & $40 \pm 11$ & $37 \pm 11$ & $43 \pm 12$ & $40 \pm 9$ & 0.91 & $<0.05$ & 0.27 \\
\hline $\operatorname{LAEF}[\%]$ & $48 \pm 11$ & $48 \pm 11$ & $25 \pm 10$ & $51 \pm 9$ & $<0.0001$ & 0.96 & 0.001 \\
\hline LA strain [\%] & $25 \pm 6$ & $27 \pm 8$ & $14 \pm 5$ & $25 \pm 7$ & $<0.001$ & 0.05 & $<0.0001$ \\
\hline RA min. volume $[\mathrm{mL}]$ & $27(23-33)$ & $24(16-28)$ & $45(34-50)$ & $33(24-34)$ & $<0.05$ & $<0.001$ & $0.01 *$ \\
\hline RA max. volume $[\mathrm{mL}]$ & $53(48-60)$ & $49(41-59)$ & $73(59-80)$ & $59(41-68)$ & 0.29 & $<0.01^{*}$ & 0.01 \\
\hline $\mathrm{RAVI}\left[\mathrm{mL} / \mathrm{m}^{2}\right]$ & $26(24-30)$ & $24(19-29)$ & $36(30-40)$ & $31(21-34)$ & 0.48 & $<0.001^{*}$ & $<0.05$ \\
\hline RA strain [\%] & $30 \pm 10$ & $36 \pm 10$ & $17 \pm 6$ & $31 \pm 10$ & 0.07 & 0.01 & $<0.01$ \\
\hline Biatrial strain [\%] & $22 \pm 9$ & $26 \pm 8$ & $10 \pm 4$ & $24 \pm 8$ & $<0.01$ & $<0.01$ & 0.0001 \\
\hline$E[\mathrm{~m} / \mathrm{s}]$ & $0.7 \pm 0.2$ & $0.7 \pm 0.2$ & $0.9 \pm 0.2$ & $0.7 \pm 0.2$ & $<0.05$ & 0.41 & 0.05 \\
\hline $\mathrm{e}^{\prime}$ mean $[\mathrm{cm} / \mathrm{s}]$ & $1 \pm 0.3$ & $1 \pm 0.3$ & $1.3 \pm 0.2$ & $0.9 \pm 0.2$ & $<0.001$ & 0.79 & $<0.001$ \\
\hline$E / e^{\prime}$ & $8 \pm 3$ & $8 \pm 3$ & $7 \pm 2$ & $7 \pm 4$ & 0.81 & 0.32 & 0.54 \\
\hline
\end{tabular}

Values are mean \pm standard deviation or median (first-third interquartile range) when normal distribution was not achieved. ^ Two-way ANOVA with replication; ${ }^{\#} \mathrm{P}$ value: t-test for related samples and where appropriate; ${ }^{*}$ Wilcoxon signed-rank test; abbreviations - see Table 2

Before RFCA mean LVEF, LV-GLS, LV size and LV wall thickness were within normal range in the present population. LV diastolic function was normal in $35(81 \%)$ patients, whereas $8(9 \%)$ patients revealed intermediate diastolic dysfunction [11]. The mean RV size and function were normal, however mean RV strain was slightly deteriorated [12]. Mean LAVI was enlarged and LA function expressed by LA strain was also impaired [13], especially in the AF group but also in the SR group. Mean RAVI was enlarged and baseline RA strain was impaired compared with healthy population [14].

Heart rate during the baseline and follow-up transthoracic examination (after $15 \pm 7$ months) was comparable. At follow-up a significant decrease in LA maximal, minimal and indexed volume was observed, however, LAVI did not normalize (did not achieve the value $\leq 34 \mathrm{~mL} / \mathrm{m}^{2}$ ). Although a substantial $4 \pm 7 \%$ improvement in LA strain was reported in the overall population, patients with baseline SR increment was only borderline sig- nificant. Regarding RA and RV size: RA minimal, maximal and indexed volume, as well as RV enddiastolic area decreased significantly.

Moreover, RA and biatrial strain showed significant improvement and increased: $8 \pm 12 \%$ ( $<<0.001), 7 \pm 10 \%$, $(\mathrm{p}<0.0001)$, respectively (Fig. 1). The RV function improved regarding RV strain (decreased $3 \pm 5 \%, \mathrm{p}<0.001$ ) and tricuspid annular plane systolic excursion by M-mode (TAPSE) $(\mathrm{p}=0.01)$.

There were no substantial differences between baseline and control parameters in LAEF, $\mathrm{LV}$ dimensions or volumes, $\mathrm{LV}$ diastolic function parameters including mitral $\mathrm{E}$ and A velocities, e', $\mathrm{E} / \mathrm{e}^{\prime}$. LVEF and LV-GLS were within normal range and showed no significant change (Table 2).

\section{Differences between groups}

with baseline SR and AF

Patients with baseline AF presented a more pronounced reduction in minimal LA volume and improvement in LA strain, LAEF and biatrial 

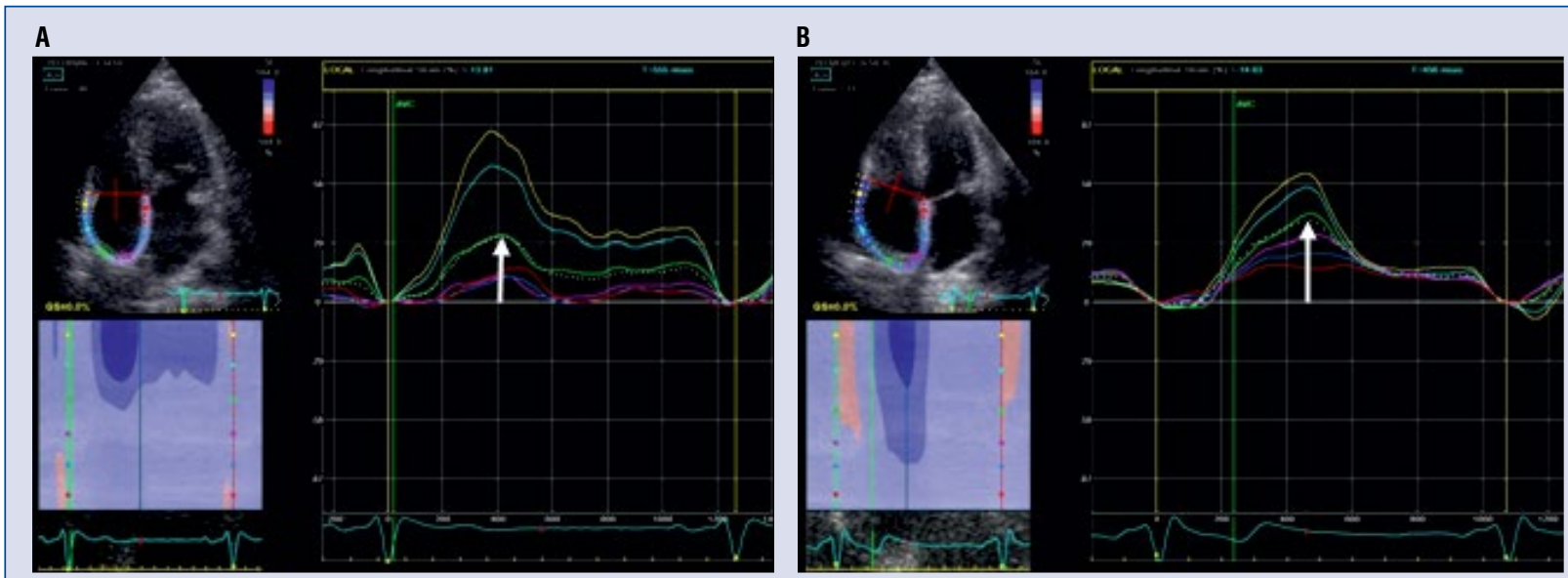

C

D
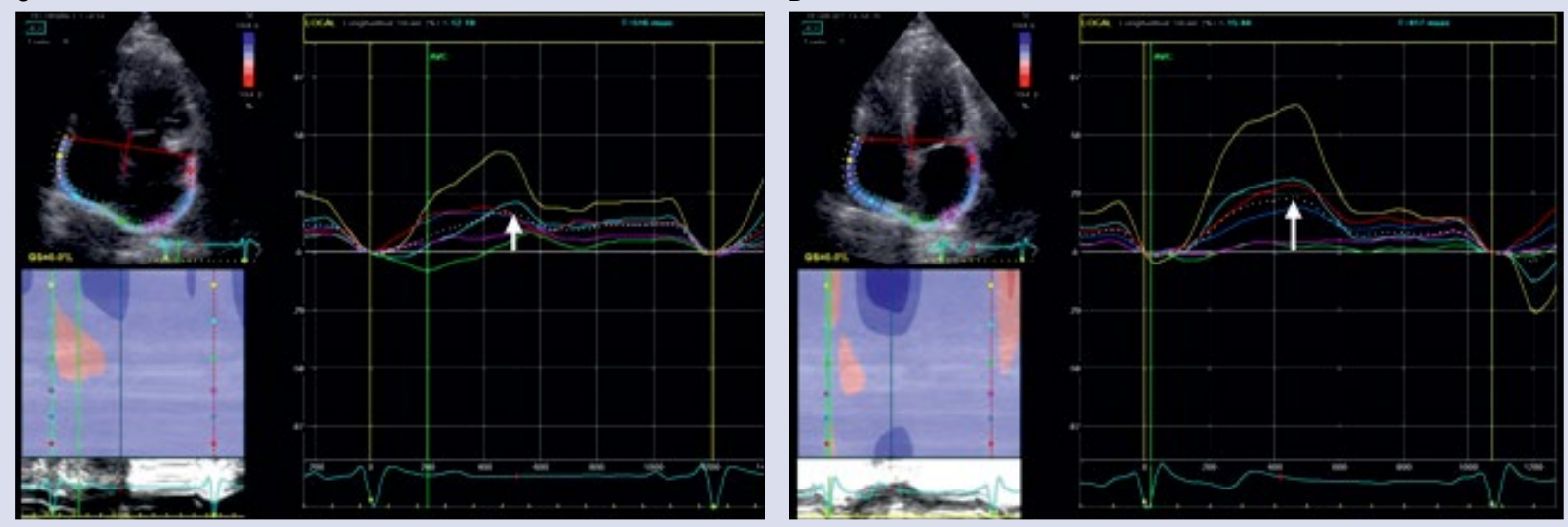

E

$\mathrm{F}$
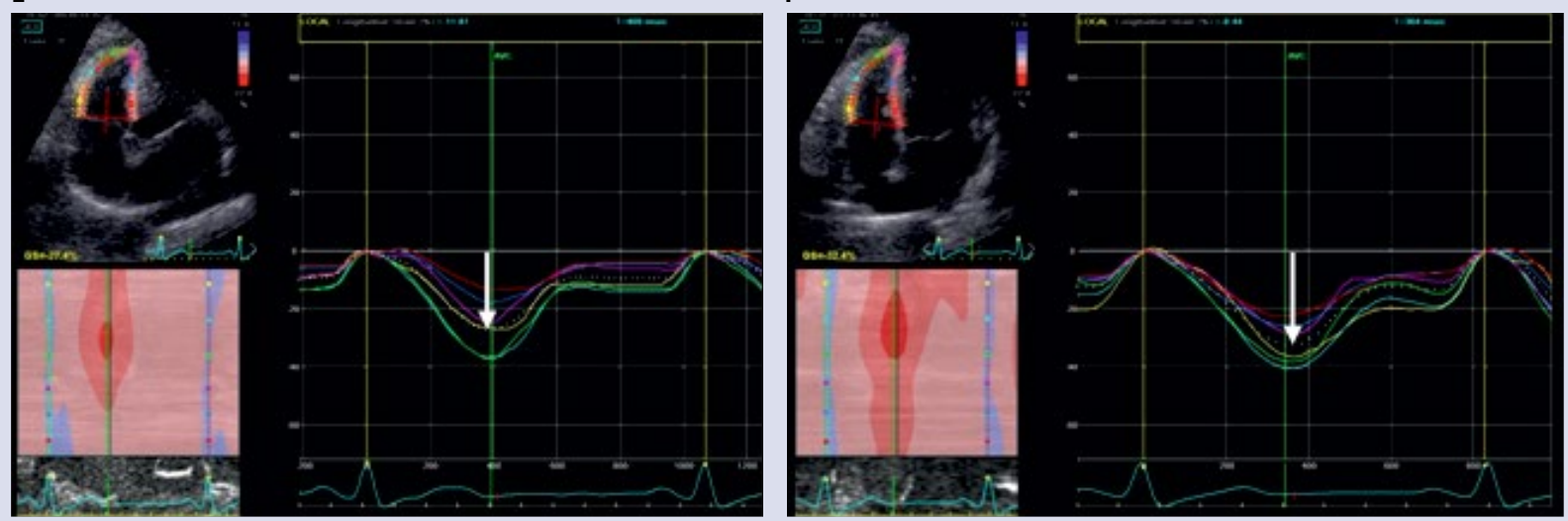

Figure 1. An example of a patient from the sinus rhythm (SR) group demonstrating improvements in right atrial (RA), biatrial strain and right ventricle (RV) strain after radiofrequency catheter ablation (RFCA). A. RA strain before: $32 \%$; B. RA strain after: $40 \%$; C. Biatrial strain before: $20 \%$; D. Biatrial strain after: $27 \%$; E. RV strain before: $-27 \%$; F. RV strain after: $-32 \%$.

strain at the follow-up examination than SR group (Fig. 2A-C). Larger improvement in RV function regarding RV strain and TAPSE in the AF group was also noticed (Fig. 2E). RA strain improved and RA volumes decreased to a similar degree in the $\mathrm{SR}$ and $\mathrm{AF}$ group (Fig. 2D). Additional substantial improvement in LVEF and GLS was noticed in the AF group (Fig. 2F, Table 3).

\section{Reproducibility}

The reproducibility of the strain measurement was good. Intra- and interobserver variability was calculated as the absolute difference (in \%) divided by the mean of the repeated measures were $2.6 \%$ and $10.8 \%$ for LA strain, $0.3 \%$ and $0.2 \%$ for RA strain, $1.5 \%$ and $4.1 \%$ for biatrial strain, $5.6 \%$ and $3.0 \%$ for RV strain and $3.6 \%$ and $2.1 \%$ for LV-GLS, respectively. 


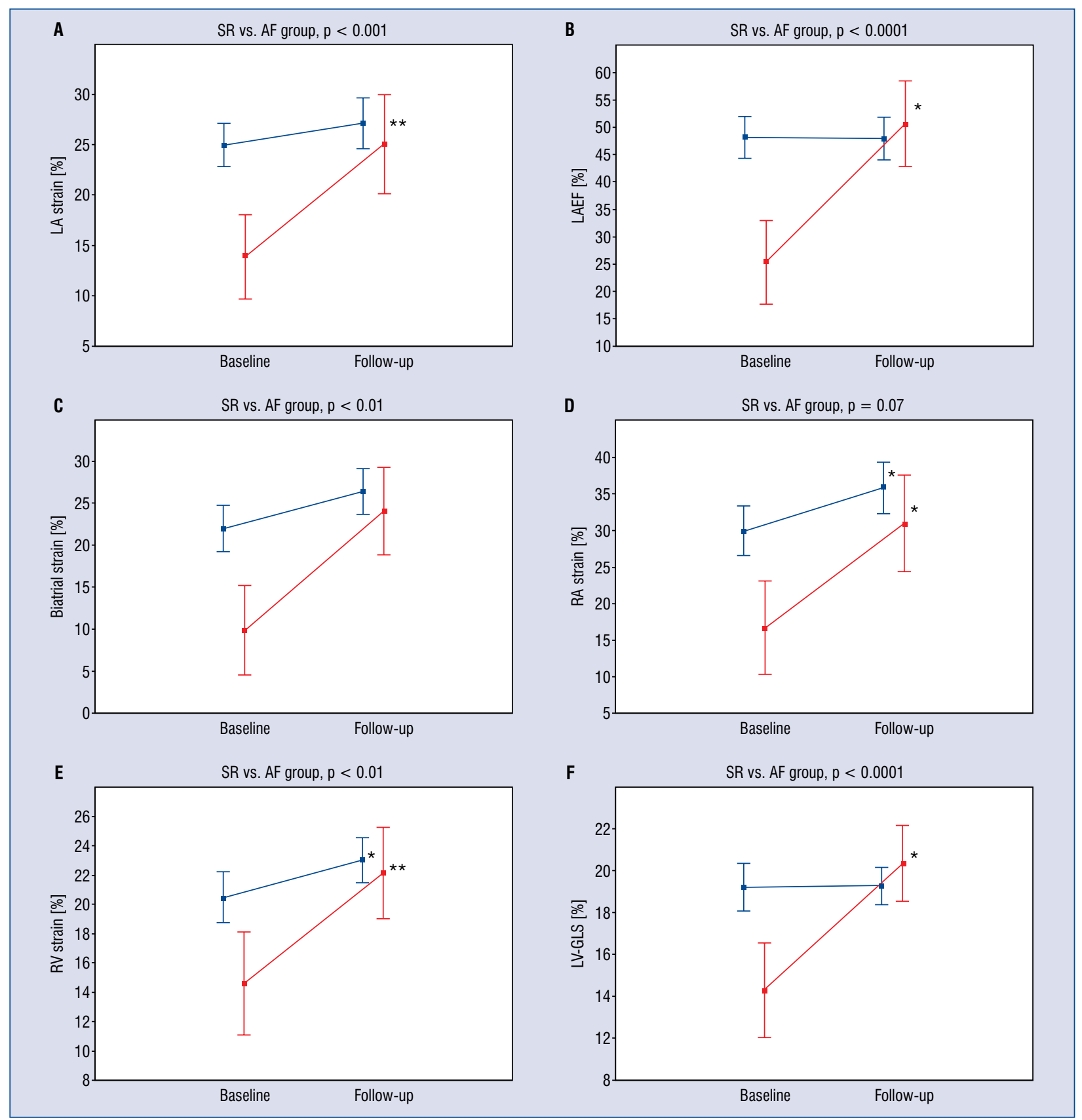

Figure 2. Changes in atrial and ventricular function according to baseline heart rhythm; A. Left atrial (LA) strain, B. LA emptying fraction (LAEF); C. Biatrial strain; D. Right atrial (RA) strain; E. Right ventricular (RV) strain; F. Left ventricular global longitudinal peak strain (LV-GLS); (-) atrial fibrillation (AF) group; (-) sinus rhythm (SR) group; SR vs. AF group - two-way ANOVA with replication; vertical bars represent 0.95 confidence intervals; baseline vs. follow-up in the AF and SR group separately - T-test for related samples ${ }^{*} p<0.01 ;{ }^{*} p<0.001$.

\section{Discussion}

The present results show a significant improvement in RA and RV function with a substantial reduction in LA and RA size after RFCA of symptomatic AF. According to available research, this is the first study to determine RA and RV function pre- and post-AF ablation using 2D-STE.
It is known that LA size, which is a surrogate measure of LA structural remodeling, decreases after $\mathrm{AF}$ ablation $[4,15,16]$. This is in agreement with the current study showing a significant reduction in the minimal, maximal and indexed LA volumes after PVI in the whole group examined.

There are still discrepancies regarding available data concerning LA function recovery after $\mathrm{AF}$ 
ablation [15, 17-19]. This inconsistency is probably the result of a variety of LA function parameters used in different studies, divergent timelines, diverse imaging techniques and finally LA measurements analyzed during AF or SR. Among the many parameters in use to assess LA performance the present study focused on LA longitudinal peak positive strain. The LA is a reservoir, a conduit and a pump, and plays an important role in modulating LV filling [10]. In order not to exclude patients with AF, QRS was chosen as the reference point. In this setting LA peak positive strain corresponds to LA reservoir function while in previous studies it was correlated with structural LA remodeling/fibrosis $[7,20]$.

The results of the present study indicate significant increases in LA strain in the overall population with no substantial change in the SR group (Fig. 2A). The improvement in LA function only in the whole group was probably the result of $\mathrm{AF}$ occurrence during baseline echocardiography in 9 patients. If baseline imaging is acquired during $\mathrm{AF}$ and the control in SR, variation of the LA strain may be overestimated.

Tops et al. [21] demonstrated significant improvement in LA strain after PVI. Contrary to the present study, the strain value was obtained by color-coded tissue Doppler imaging in patients with a relatively small baseline LAVI compared with population herein $\left(30 \pm 7\right.$ vs. $\left.41 \pm 12 \mathrm{~mL} / \mathrm{m}^{2}\right)$. Additionally, it is unknown how many patients had $\mathrm{AF}$ during echocardiographic examination before PVI.

In agreement the present SR group results, another study [22] also showed no significant change in LA strain pre- and post-PVI in patients with paroxysmal and persistent AF. However, the authors did not provide information about patient heart rhythm during image acquisition.

Finally, regarding LAEF, no substantial change was seen in the overall population or in the SR group (baseline LAEF was $>40 \%$ in both groups). This is in agreement with Antolini et al. [19] who reported that only patients with baseline LAEF $<40 \%$ had significant improvement in LA performance after ablation.

Santoro et al. [23] showed that uncontrolled hypertension confers higher $\mathrm{AF}$ recurrence risk after RFCA and can affect outcome regarding LA diameter and scar extension. Patients from the current study, however, had well controlled blood pressure with drugs, so it was believed that the influence of hypertension on LA strain values was minimal [23].

In sum, PVI exerts a beneficial effect on LA hemodynamics. However, despite LA volume de- cline, only minor improvement in LA function was observed. These discrepancies can be explained by the fact that changes in LA function after AF ablation depend on the balance between the beneficial effect of atrial reverse remodeling after SR restoration and the harmful effect of fibrosis and edema caused by the ablation procedure.

In agreement with a previous series the present study shows a significant reduction in RA size after PVI [24, 25], which is probably a result of SR restoration. It was also shown that changes in LA and RA volume were significantly correlated and reverse remodeling was faster in the RA than in the LA $[24,26]$. It is known, that cavo-tricuspid isthmus ablation in RA may lead, per se, to volume reduction via the scaring process. Regarding the current study however, only 1 patient underwent such ablation.

According to available research, an improvement in the RA function in terms of RA strain is presently reported for the first time. In contrast to LA strain, RA strain improved both in the SR and $\mathrm{AF}$ group, however to a higher degree in the $\mathrm{AF}$ group. This observation is in agreement with Wylie et al. [25], who reported a significant increase in RAEF after AF ablation. However, the analysis was based on 33 patients who underwent PVI of which 20 also had a cavo-tricuspid isthmus block line created due to atrial flutter. According to all available research, the impact of RFCA on RV function remains unknown. The present data showed significant improvement in RV function (expressed by an increase in TAPSE and RV strain) with concomitant RV end diastolic area reduction. Probably, an improvement in RA function ultimately results in favorable RV reverse remodeling and vice versa. There are a lack of studies to compare these results to and thus, it is believed that this will encourage further investigation.

No significant improvement in LVEF was observed in the current analysis in patients with LVEF $\geq 50 \%$. LV-GLS revealed borderline improvement in the overall population $(\mathrm{p}=0.05)$ with significant improvement in the AF group and no change in the SR group. LV-GLS is a very sensitive parameter and in spite of almost normal LVEF in the AF group ( $\geq 50 \%$ ) during baseline echocardiographic examination, LV-GLS was already substantially impaired. Previous studies have demonstrated a beneficial effect of AF ablation on LV function in patients with heart failure and impaired LVEF $[3,4]$. Nevertheless, in patients with preserved LVEF the effect of RFCA on LV function was considered less favorable as EF remained unchanged after 
ablation [5]. Subtle changes in LV however, may not be detected using conventional methods. Indeed, preliminary data in patients with preserved LVEF show that LV deformation may improve after $\mathrm{AF}$ ablation. Previous studies revealed a significant increase in the absolute values of circumferential and LV-GLS, particularly in patients who maintained SR after PVI [6, 27]. Furthermore Lin et al. [28] described subtle LV changes using another echocardiographic method - vector flow mapping, evaluating LV energy loss. This parameter, as a marker of structural LV remodeling, almost normalized at 6 months follow-up after successful RFCA. Similarly, substantial improvement of native T1 mapping assessed by cardiac magnetic resonance - a marker of reverse LV remodeling - was observed only in patients with successfully restored SR following RFCA [29]. All of the abovementioned studies, found significant $\mathrm{LV}$ improvement in patients with preserved LVEF only after successful RFCA. After ablation, SR maintained $74 \%$ in the present study population.

According to available research, this is the first study that assesses changes in biatrial strain before and after PVI (Fig. 1C, D). In this study biatrial strain showed significant improvement regardless of the rhythm during baseline examination. A considered opinion herein posits that the evaluation of atrial free wall strain can more precisely assess true atrial remodeling due to the avoidance of artifactual dropout of the atrial septum. In addition, this parameter can be a common thread regarding atrial assessment as changes in LA and RA volume are significantly correlated [23].

\section{Difference between the SR group and group with baseline $\mathrm{AF}$}

The baseline AF group showed more pronounced improvement in cardiac function and greater LA minimal and RA volume decrease than SR group. This may be explained by several hypotheses. First, persistent AF was significantly more frequent in the AF group, and PVI is probably more effective regarding reverse remodeling in patients with persistent AF. Second, a significant increase in heart rate was found in the SR and a decrease in the $\mathrm{AF}$ group between the baseline and control echocardiographic examination (Table 3). Therefore, improvement in cardiac function after $\mathrm{RFCA}$ in the $\mathrm{AF}$ group could be more related to normalization of heart rate than to maintenance of $\mathrm{SR}$. Finally, measurements in the AF group were collected during AF before RFCA and SR at followup. Thus, variations of functional and volumetric parameters could have been overestimated.

\section{Limitations of the study}

First, the low sample size and relatively short follow up are limitations of the current analysis. Second, although 2D-STE software is primarily designed for LV analysis, it was used for atrial and RV deformation measurements, having said that however, reproducibility of the measurements is good. Third, the differences between patients with AF recurrences (11 patients) and patients with maintenance of SR after RFCA were not analyzed. Finally, some patients had a discordant rhythm during baseline and follow-up image acquisition and this could have influenced comparisons of echocardiographic parameters.

\section{Conclusions}

The present study used 2D-STE showing that $\mathrm{AF}$ ablation induces favorable structural and functional remodeling mainly within the RA and RV. PVI reduces LA volume without a deleterious impact on its function. The improvement is higher in patients with AF during baseline examination. Present observations may further encourage physicians to consider ablation in relatively young, healthy patients with AF and without obvious structural heart disease in order to reverse the $\mathrm{AF}$ induced incipient cardiomyopathy and restore normal cardiac function.

\section{Conflict of interest: None declared}

\section{References}

1. Kirchhof P, Benussi S, Kotecha D, et al. ESC Scientific Document Group. 2016 ESC Guidelines for the management of atrial fibrillation developed in collaboration with EACTS. Eur Heart J. 2016; 37(38): 2893-2962, doi: 10.1093/eurheartj/ehw210, indexed in Pubmed: 27567408.

2. Ejima K, Kato K, Arai K, et al. Impact of atrial remodeling on the outcome of radiofrequency catheter ablation of paroxysmal atrial fibrillation. Circ J. 2014; 78(4): 872-877, indexed in Pubmed: 24562637.

3. Marrouche N, Brachmann J, Andresen D, et al. Catheter ablation for atrial fibrillation with heart failure. N Engl J Med. 2018; 378(5): 417-427, doi: 10.1056/nejmoa1707855.

4. Calkins H, Hindricks G, Cappato R, et al. 2017 HRS/EHRA/ ECAS/APHRS/SOLAECE expert consensus statement on catheter and surgical ablation of atrial fibrillation. EP Europace. 2017; 20(1): e1-e160, doi: 10.1093/europace/eux274.

5. Lutomsky BA, Rostock T, Koops A, et al. Catheter ablation of paroxysmal atrial fibrillation improves cardiac function: a prospective study on the impact of atrial fibrillation ablation on left ventricular function assessed by magnetic resonance imaging. Europace. 2008; 10(5): 593-599, doi: 10.1093/europace/eun076, indexed in Pubmed: 18385123. 
6. Tops LF, Den Uijl DW, Delgado V, et al. Long-term improvement in left ventricular strain after successful catheter ablation for atrial fibrillation in patients with preserved left ventricular systolic function. Circ Arrhythm Electrophysiol. 2009; 2(3): 249-257, doi: 10.1161/CIRCEP.108.838748, indexed in Pubmed: 19808475.

7. Kuppahally SS, Akoum N, Burgon NS, et al. Left atrial strain and strain rate in patients with paroxysmal and persistent atrial fibrillation: relationship to left atrial structural remodeling detected by delayed-enhancement MRI. Circ Cardiovasc Imaging. 2010; 3(3): 231-239, doi: 10.1161/CIRCIMAGING.109.865683, indexed in Pubmed: 20133512.

8. Cameli M, Lisi M, Righini FM, et al. Usefulness of atrial deformation analysis to predict left atrial fibrosis and endocardial thickness in patients undergoing mitral valve operations for severe mitral regurgitation secondary to mitral valve prolapse. Am J Cardiol. 2013; 111(4): 595-601, doi: 10.1016/j.amjcard.2012.10.049, indexed in Pubmed: 23211360.

9. Lang R, Badano L, Mor-Avi V, et al. Recommendations for Cardiac Chamber Quantification by Echocardiography in Adults: An Update from the American Society of Echocardiography and the European Association of Cardiovascular Imaging. Eur Heart J Cardiovasc Imaging. 2015; 16(3): 233-271, doi: 10.1093/ehjci/ /jev014.

10. Badano LP, Kolias TJ, Muraru D, et al. Standardization of left atrial, right ventricular, and right atrial deformation imaging using two-dimensional speckle tracking echocardiography: a consensus document of the EACVI/ASE/Industry Task Force to standardize deformation imaging. Eur Heart J Cardiovasc Imaging. 2018; 19(6): 591-600, doi: 10.1093/ehjci/jey042, indexed in Pubmed: 29596561.

11. Nagueh S, Smiseth O, Appleton C, et al. Recommendations for the Evaluation of Left Ventricular Diastolic Function by Echocardiography: An Update from the American Society of Echocardiography and the European Association of Cardiovascular Imaging. Eur J Echocardiogr. 2016; 17(12): 1321-1360, doi: 10.1093/ ehjci/jew082.

12. Muraru D, Onciul S, Peluso D, et al. Sex- and method-specific reference values for right ventricular strain by 2-dimensional speckle-tracking echocardiography. Circ Cardiovasc Imaging. 2016; 9(2): e003866, doi: 10.1161/CIRCIMAGING.115.003866, indexed in Pubmed: 26860970.

13. Pathan F, D'Elia N, Nolan MT, et al. Normal ranges of left atrial strain by speckle-tracking echocardiography: a systematic review and meta-analysis. J Am Soc Echocardiogr. 2017; 30(1): 59-70.e8, doi: 10.1016/j.echo.2016.09.007, indexed in Pubmed: 28341032.

14. Padeletti M, Cameli M, Lisi M, et al. Reference values of right atrial longitudinal strain imaging by two-dimensional speckle tracking. Echocardiography. 2012; 29(2): 147-152, doi: 10.1111/j.15408175.2011.01564.x, indexed in Pubmed: 22118219.

15. Jeevanantham V, Ntim W, Navaneethan SD, et al. Meta-analysis of the effect of radiofrequency catheter ablation on left atrial size, volumes and function in patients with atrial fibrillation. Am J Cardiol. 2010; 105(9): 1317-1326, doi: 10.1016/j.amjcard.2009.12.046, indexed in Pubmed: 20403486.

16. Xiong B, Li D, Wang J, et al. The effect of catheter ablation on left atrial size and function for patients with atrial fibrillation: an updated meta-analysis. PLoS One. 2015; 10(7): e0129274, doi: 10.1371/journal.pone.0129274, indexed in Pubmed: 26147984 .
17. Pump A, Di Biase L, Price J, et al. Efficacy of catheter ablation in nonparoxysmal atrial fibrillation patients with severe enlarged left atrium and its impact on left atrial structural remodeling. J Cardiovasc Electrophysiol. 2013; 24(11): 1224-1231, doi: 10.1111/jce.12253, indexed in Pubmed: 24020717.

18. Montserrat S, Sitges M, Calvo N, et al. Effect of repeated radiofrequency catheter ablation on left atrial function for the treatment of atrial fibrillation. Am J Cardiol. 2011; 108(12): 1741-1746, doi: 10.1016/j.amjcard.2011.07.041, indexed in Pubmed: 21906703.

19. Antolini M, Brustio A, Morello M, et al. Left atrial function after radiofrequency catheter ablation of atrial fibrillation -- can pre-ablation function predict contractile improvement during follow-up? Circ J. 2015; 79(12): 2576-2583, doi: 10.1253/circj. CJ-15-0184, indexed in Pubmed: 26447119.

20. Habibi M, Lima JAC, Khurram IM, et al. Association of left atrial function and left atrial enhancement in patients with atrial fibrillation: cardiac magnetic resonance study. Circ Cardiovasc Imaging. 2015; 8(2): e002769, doi: 10.1161/CIRCIMAGING.114.002769, indexed in Pubmed: 25652181.

21. Tops LF, Delgado V, Bertini M, et al. Left atrial strain predicts reverse remodeling after catheter ablation for atrial fibrillation. J Am Coll Cardiol. 2011; 57(3): 324-331, doi: 10.1016/j. jacc.2010.05.063, indexed in Pubmed: 21232671.

22. Kawakami H, Inoue K, Fujii A, et al. Evaluation of functional recovery of structural reverse remodeling of the left atrium by pulmonary vein isolation in patients with atrial fibrillation. Eur Heart J. 2018; 39(suppl_1): P5355, doi: 10.1093/eurheartj/ ehy566.p5355.

23. Santoro F, Di Biase L, Trivedi C, et al. Impact of uncontrolled hypertension on Atrial fibrillation ablation outcome. JACC Clin Electrophysiol. 2015; 1(3): 164-173, doi: 10.1016/j.jacep.2015.04.002, indexed in Pubmed: 29759360.

24. Müller H, Noble S, Keller PF, et al. Biatrial anatomical reverse remodelling after radiofrequency catheter ablation for atrial fibrillation: evidence from real-time three-dimensional echocardiography. Europace. 2008; 10(9): 1073-1078, doi: 10.1093/ europace/eun187, indexed in Pubmed: 18628258.

25. Wylie JV, Peters DC, Essebag V, et al. Left atrial function and scar after catheter ablation of atrial fibrillation. Heart Rhythm. 2008; 5(5): 656-662, doi: 10.1016/j.hrthm.2008.02.008, indexed in Pubmed: 18452866.

26. Lo LW, Chen SA. Cardiac remodeling after atrial fibrillation ablation. J Atr Fibrillation. 2013; 6(1).

27. Machino-Ohtsuka T, Seo Y, Ishizu T, et al. Efficacy, safety, and outcomes of catheter ablation of atrial fibrillation in patients with heart failure with preserved ejection fraction. J Am Coll Cardiol. 2013; 62(20): 1857-1865, doi: 10.1016/j.jacc.2013.07.020, indexed in Pubmed: 23916940.

28. Lin M, Hao Li, Cao Y, et al. Successful radiofrequency catheter ablation of atrial fibrillation is associated with improvement in left ventricular energy loss and mechanics abnormalities. Int J Cardiovasc Imaging. 2018 [Epub ahead of print], doi: 10.1007/ s10554-018-1457-3, indexed in Pubmed: 30293193.

29. Kowallick JT, Staab W, Schuster A, et al. Reverse left ventricular structural remodeling after catheter ablation of atrial fibrillation in patients with preserved left ventricular function: Insights from cardiovascular magnetic resonance native T1 mapping. Heart Rhythm. 2019; 16(3): 424-432, doi: 10.1016/j.hrthm.2018.09.016, indexed in Pubmed: 30248459. 